\title{
Identification, Modeling and Control of Unmanned Aerial Vehicles
}

\author{
Nilesh Kumar and Sheilza Jain \\ Department of Electronics Engineering \\ YMCA University of Science and Technology (Faridabad) Haryana, India \\ nileshkumar_yadav@yahoo.co.in
}

\begin{abstract}
This paper presents a method to control height and speed of fixed wing unmanned aerial vehicles (UAVs) using MATLAB platform. The mathematical dynamic model of Six Degrees of Freedom (6-DOF) aircraft is nonlinear and it is linearized about a flight condition. System is divided into two Multi Input Multi Output (MIMO) sub models. First model controls the longitudinal dynamics and second model is used to control the lateral dynamics. Each MIMO model is converted into ten Single Input Single Output (SISO) systems and then the systems needed for the primary requirement of plant control is selected. Same technique can be also be used to make Manned Aerial Vehicles (MAV) autopilots and to control flight of other aerial vehicles. Multiple loop feedback control technique is used to control a plant which helps to control many outputs of system with one set point value and controller.
\end{abstract}

Keywords: Unmanned Aerial Vehicle, Flight Control, Longitudinal Autopilot, MIMO, SISO

\section{Introduction}

At present, the use of advance control systems is increasing day by day in every field; aeronautics is one out of them. From generations, it was a desire of human to develop intelligent systems which can obey them. Unmanned aerial vehicles are one such development of aeronautical, instrumentation and control system technologies [1]. UAVs have a wide range of civil and military applications and are of different sizes according to applications [2].

Remote sensing, Commercial aerial surveillance, Archaeology, Forest fire detection, Armed attacks, research, Oil, Commercial and Motion Picture Filmmaking, Search and rescue operations, Maritime patrol and Aerial target practice in training of human pilots are the few out of many applications where UAVs have proved to be an alternative and fill the gap where the risk of human piloted aircraft is not acceptable or impractical. The main building block of UAV is its automatic flight controller which is known as autopilot [3].This subsystem controls UAV by generating control signals on the basis of desired target information and waypoints.

Various sensors are used in UAVs to generate feedback signals for the controller on the basis of real time state of vehicle. The autopilot is controlled by a pilot on the ground or by another vehicle. A typical launch and recovery method of an unmanned aircraft is done by an automatic system or an operator on the ground. The traditional approach used for flight control system synthesis, implementation and validation is time consuming and resource intensive. Applying the same techniques for medium and small vehicles is not realistic. To make cost effective autopilots for these aerial vehicles, 
computer technology plays an important role. MATLAB is one such platform on which it is possible to simulate and to test the performance of an autopilot. Conventional PI (Proportional Integral) and PID (Proportional Integral Derivative) controllers can be used to control autopilots on this platform and hence to improve their performance characteristics.

\section{Identification and Modeling of UAV}

A 6-DOF medium sized, fixed wing UAV's mathematical model is multivariable, time variant and non-linear in nature. Understanding and solving such a plant is very complex. So, to convert non-linear model of UAV into a linearized model is the primary requirement for autopilot designing which provides clear insight into the system dynamics.

The concept of mathematical model used in this paper is derived from a mathematical model used for fixed wing, medium sized unmanned aerial vehicle. Its small perturbation linearized model is adapted to design autopilots for height, sideslip and speed control of UAV. The schematic of UAV with North-East-Down (NED) axis is shown in Figure 1.

The nonlinear dynamic model is developed by the help of force and moment equations. An aircraft has three linear and three rotational motions to compose its 6DOF. Laws of physics can be applied on the vehicle to find mathematical differential equations. All the calculations are done by taking parameter values with respect to center of gravity of vehicle. All forces and moments depend on the thrust applied by the engine and engine speed is taken in rpm (revolution per minute)

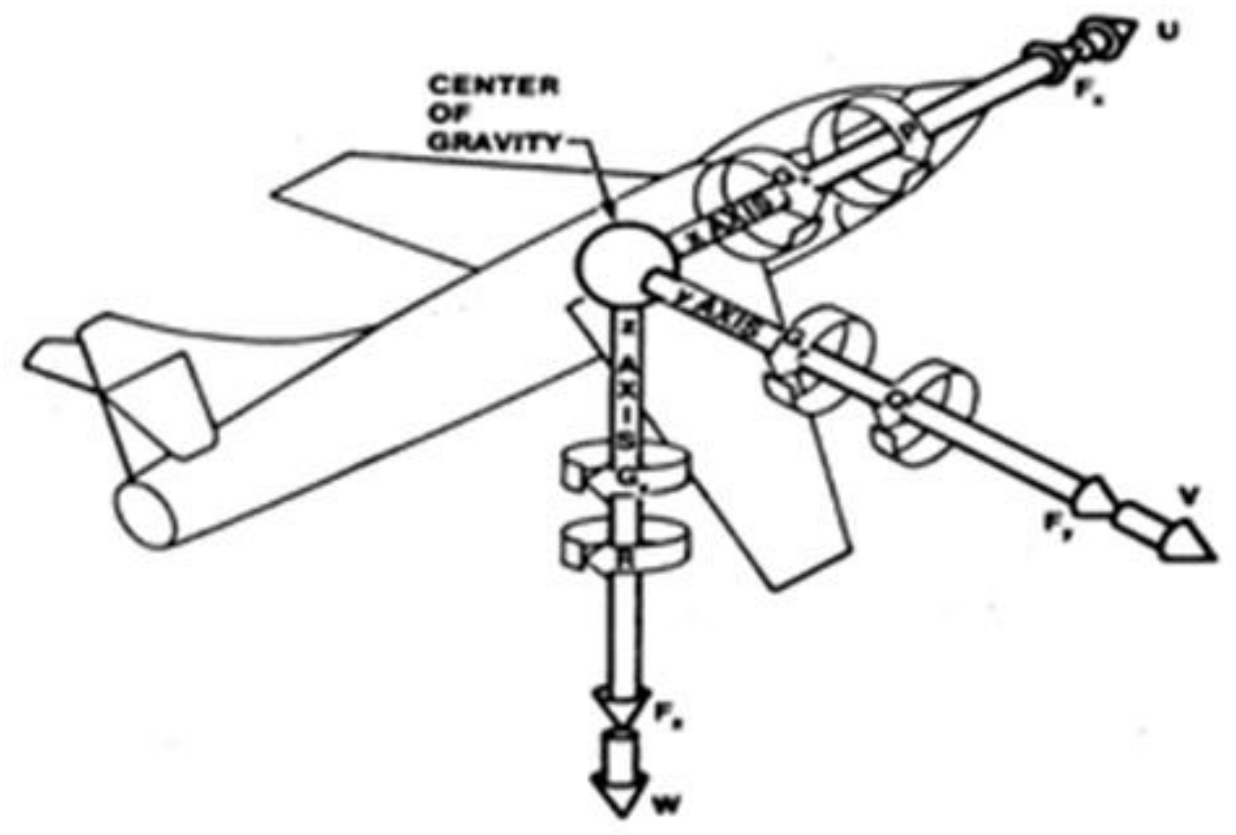

Figure 1. UAV Fixed NED Axis System and Notation 


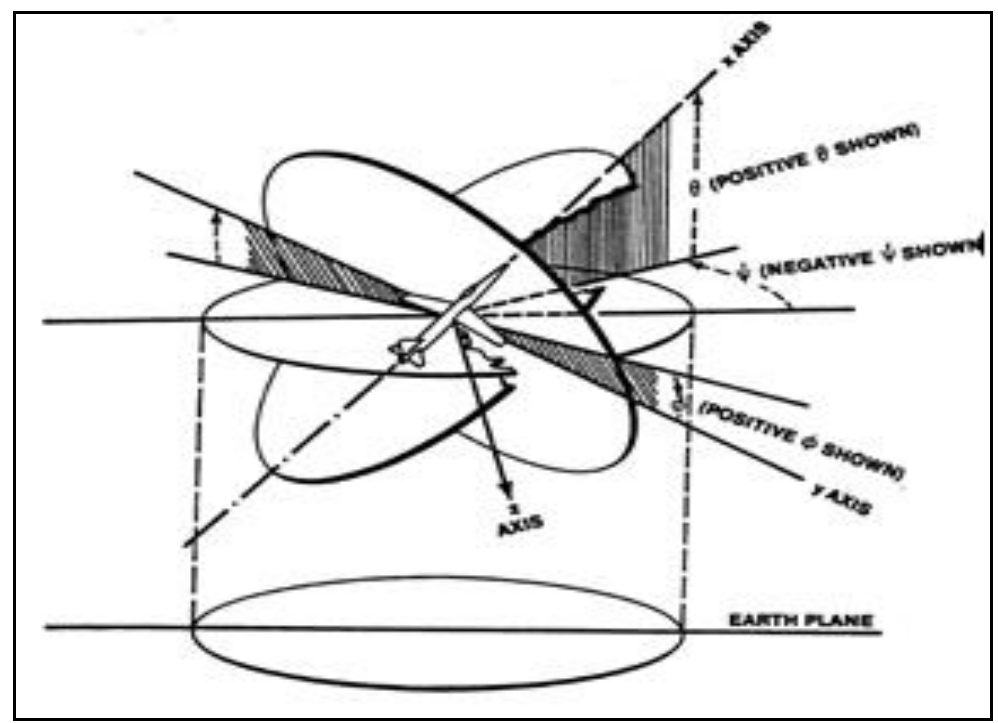

Figure 2. Euler Angles Rotations and Notations of UAV

Consider vehicle heading along $\mathrm{x}$ axis, its right wing along $\mathrm{y}$ axis and $\mathrm{z}$ axis downward which passes through the center of gravity of vehicle and perpendicular to both $\mathrm{x}$ and $\mathrm{y}$ axis as shown in Figure 2. Suppose the state vectors are in north east down projection.

By assuming flat earth model, the nonlinear dynamic state equations of UAVs are [5, 6]:

$$
\begin{aligned}
& \dot{U}=R V-Q W-g_{D} \sin \theta+\left(X_{A}+X_{T}\right) / M \\
& \dot{V}=-R U+P W+g_{D} \sin \emptyset \cos \theta+\left(Y_{A}+Y_{T}\right) / M \\
& \dot{W}=Q U-P V+g_{D} \cos \emptyset \cos \theta+\left(Z_{A}+Z_{T}\right) / M \\
& \dot{\emptyset}=P+\tan \theta(Q \sin \emptyset+R \cos \emptyset) \\
& \dot{\theta}=Q \cos \emptyset-R \sin \emptyset \\
& \dot{\psi}=(Q \sin \phi+R \cos \phi) / \cos \theta \\
& \Gamma \dot{P}=J_{X Z}\left[J_{X}-J_{Y}+J_{Z}\right] P Q-\left[J_{Z}\left(J_{Z}-J_{Y}\right)+J_{X Z}^{2}\right] Q R+J_{Z} \ell+J_{X Z} n \\
& I_{Y} \dot{Q}=\left(J_{Z}-J_{X}\right) P R-J_{X Z}\left(P^{2}-R^{2}\right)+m \\
& \Gamma \dot{R}=\left[\left(J_{X}-J_{Y}\right) J_{X}-J_{X Z}^{2}\right] P Q-J_{X Z}\left[J_{X}-J_{Y}+J_{Z}\right] Q R+J_{X Z} \ell+J_{X} n \\
& \dot{p}_{N}=U c \theta c \psi+V(-c \phi \psi+s \phi s \theta c \psi)+W(s \phi s \psi+c \phi s \theta c \psi) \\
& \dot{p}_{E}=U c \theta s \psi+V(c \phi c \psi+s \phi s \theta s \psi)+W(-s \phi c \psi+c \phi s \theta s \psi) \\
& \dot{h}=U s \theta-V s \phi c \theta-W c \phi c \theta
\end{aligned}
$$


Where

$X=\left[p_{N} p_{E} p_{D} \phi \theta \psi U V W P Q R\right],\left[X_{T}, Y_{A}, Z_{A}\right]$ Are aerodynamic forces along $\mathrm{x}, \mathrm{y}$ and $\mathrm{z}$ axis and $\left[\ell_{x} \mathrm{~m}, \mathrm{n}\right]$ are moments along $\mathrm{x}, \mathrm{y}$ and $\mathrm{z}$ axis. The propulsive forces are represented by $\left[X_{T}, Y_{T}, Z_{T}\right]$.

$\Gamma=J_{X} J_{Z}-J_{X Z}^{2}$, where $\mathrm{J}$ represents the $3 \times 3$ inertia matrix.

$\theta, \phi$ and $\psi$ are the Euler angles (roll, yaw and pitch angles). These aerodynamic forces and moments depends on elevator, throttle, aileron and rudder actuator control vectors $\left[\delta_{e}, \delta_{t}, \delta_{a}, \delta_{r}\right]$ and various other flight variables. $g_{D}$ is acceleration due to gravity in North-East-Down (NED) axis and $\mathrm{h}=-p_{D}$ with vehicle weight $\mathrm{M}$.

$c \phi=\cos \phi$ and $s \phi=\sin \phi$ are the other notations used in above written equations. Decoupling of nonlinear equations is done by considering both bank angle and sideslip angle equal to zero which provides a normal flight condition and convert nonlinear set of equations into two sets for longitudinal and lateral motions of aircraft [7]. Conversion of nonlinear equations into linear equations uses small perturbation theory at normal non accelerated flight at constant speed. Nonlinear model can be linearized by finding equilibrium state or trim condition of the vehicle using MATLAB environment.

\section{State Space Model of Longitudinal Parameters}

State space model of MIMO Longitudinal subsystem represented by matrices A, B, C and $\mathrm{D}$ is converted into SISO transfer functions by using MATLAB commands. Four out of ten SISO transfer functions are chosen which have the input - output relationship between 'Elevator and q, theta, height' and 'Throttle and speed' of aircraft.

State space model of longitudinal parameters of UAV can be described by state and output equation [8] given as

$$
\begin{aligned}
& \dot{x}=A x+B u \\
& y=C x+D u
\end{aligned}
$$

$$
\begin{aligned}
& \text { The state space matrices } \mathrm{A}, \mathrm{B}, \mathrm{C} \text { and } \mathrm{D} \text { of UAV are } \\
& \mathrm{A}=\left[\begin{array}{ccccc}
-0.0844 & 0.4354 & -4.3589 & -9.7483 & 0 \\
-0.2920 & -1.8188 & 39.7431 & -1.0682 & 0 \\
0.0313 & -0.3089 & -2.3089 & -2.3953 & 0 \\
0 & 0 & 1 & 0 & 0 \\
0.1090 & -0.9940 & 0 & 40.9624 & 0
\end{array}\right] \\
& \mathrm{B}=\left[\begin{array}{cc}
-0.0494 & 144.8262 \\
-3.2438 & 0 \\
8.6497 & -7.2413 \\
0 & 0 \\
0 & 0
\end{array}\right] \\
& \mathrm{C}=\left[\begin{array}{cccc}
1 & 00 & 0 & 0 \\
0 & 10 & 0 & 0 \\
0 & 01 & 0 & 0 \\
0 & 00 & 1 & 0 \\
0 & 00 & 0 & 1
\end{array}\right] ; \quad \mathrm{D}=\left[\begin{array}{cc}
0 & 0 \\
0 & 0 \\
0 & 0 \\
0 & 0 \\
0 & 0
\end{array}\right]
\end{aligned}
$$


Where; A, B, C and D are the state matrices. Input $u=\left[\delta_{e} \delta_{t h}\right]^{T}$ and states $x=[\mathrm{u} \text { w q } \theta \mathrm{h}]^{T}$. Output vector $y$ also consists the states of the system.

The SISO transfer functions can be controlled by appropriate P (proportional), PI or PID controllers [9, 10]. Open loop Pole- Zero locations can be obtained and effect of gain on stability of system can be observed by root locus plot. Gain Margin and Phase Margin can also be determined for relative stability analysis of the system.

\section{Dynamics of Open Loop Longitudinal Subsystem of UAV}

Longitudinal Subsystem can be represented by various transfer functions of UAV with set point values and outputs. Feedback loop and controller are not required in open loop system. Unit-step test signal is taken as set point value for the system and all the responses are observed corresponding to this step input $[11,12]$. The simulated diagram of open loop longitudinal system using MATLAB environment is shown in Figure 3.

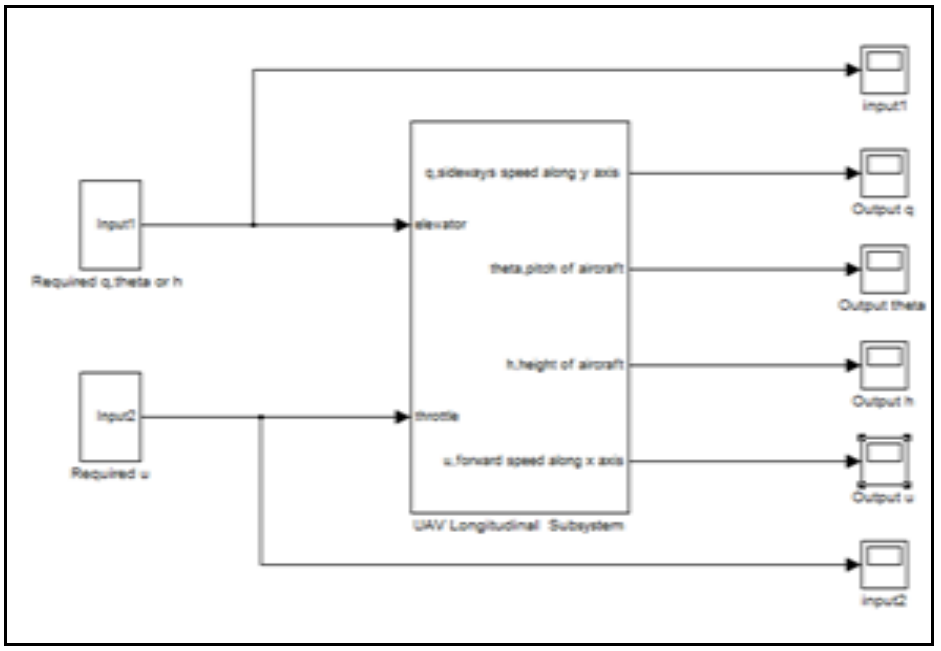

Figure 3. Simulation of Open Loop Longitudinal System

The open loop sideslip response, height response and speed response of UAV are given in Figures 4, 5 and 6 respectively.

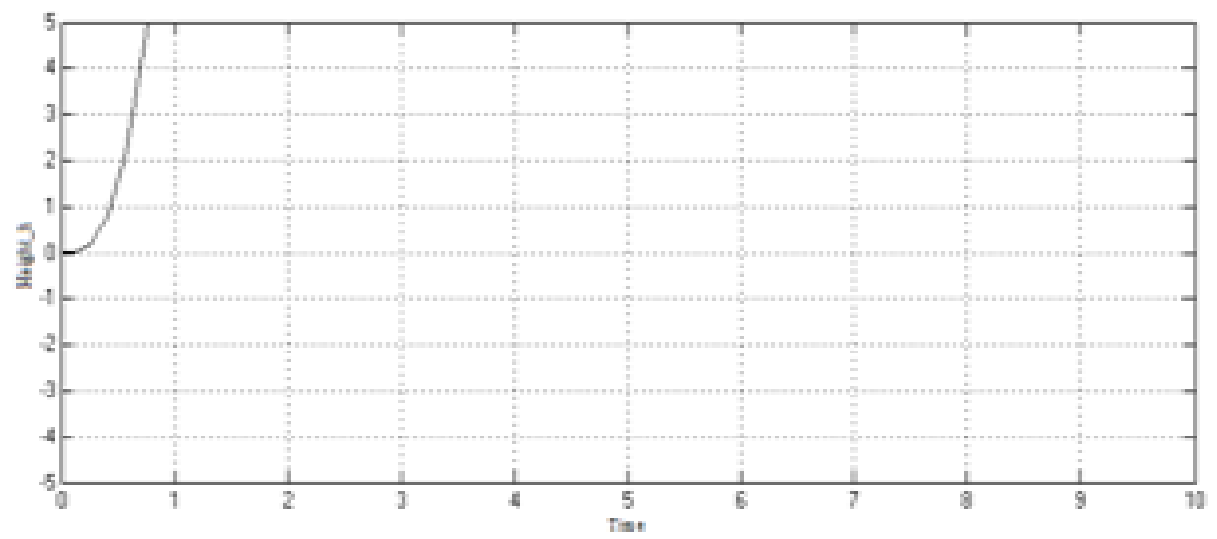

Figure 4. Open Loop Sideslip Response of UAV 


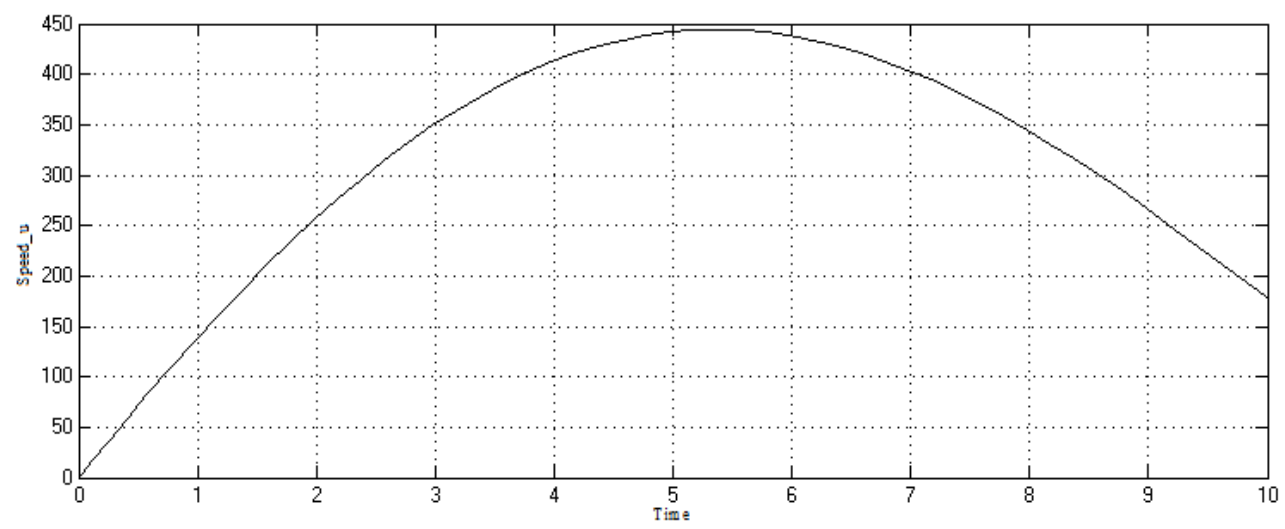

Figure 5. Open Loop Height Response of UAV

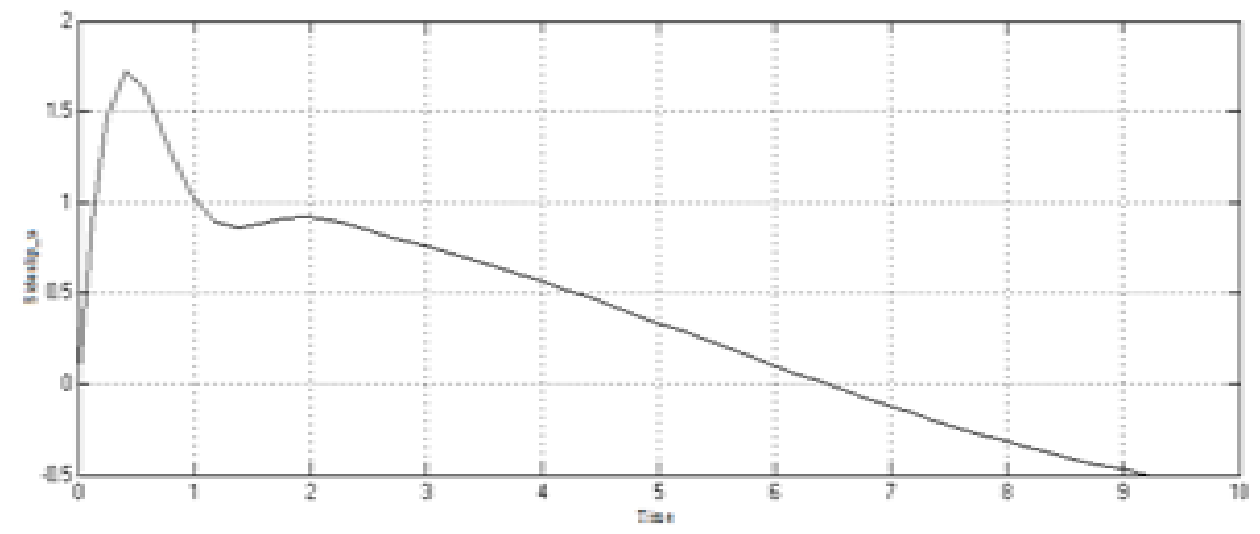

Figure 6. Open Loop Speed Response of UAV

\section{Closed Loop Control of UAV}

Linear model of UAV consists of longitudinal and lateral model of system. Control of height, speed and sideslip of aerial vehicle requires only longitudinal model of the aircraft having five states and two inputs i.e., Elevator and Throttle [13,14]. Elevator is used to control height, pitch, pitch rate and sideslip of the aircraft while Throttle is used to control vehicle speed.

\section{Closed Loop Design Structure}

The major task for the control of longitudinal parameter of the plant is:

a) Control of Elevator deflection for Height control

b) Control of Engine Throttle for speed control

The guidance block of the vehicle generating commands for height, sideslip and speed control are based on the required trajectory. These control objectives are achieved by converting MIMO problem into SISO problems [15]. Multiple loop feedback technique is used to control SISO systems. Control of height and pitch is done by applying multiple feedbacks and control of sideslip and speed is done by individual feedback loops [16]. The Simulink diagram of sideslip and speed control and Simulink 
diagram for height and speed control of UAV are shown in Figures 7 and 8 respectively.

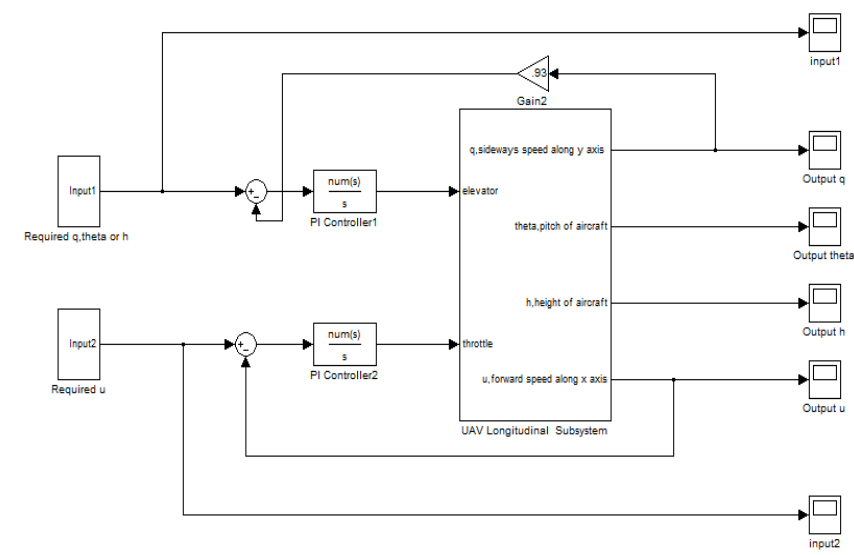

Figure 7. Simulink Diagram for Sideslip \& Speed Control

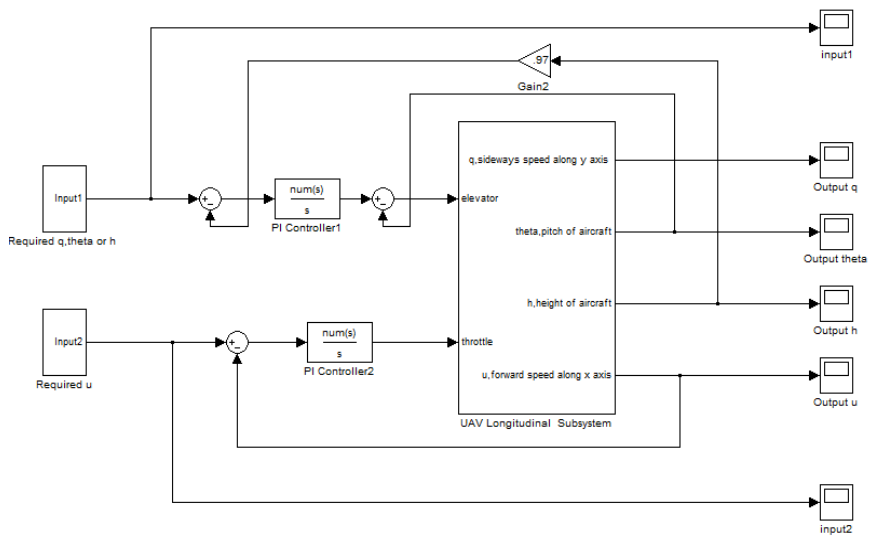

Figure 8. Simulink Diagram for Height \& Speed control

5.1. Design of Height Control Feedback Loop: To design height control feedback loop, MIMO system is converted into SISO systems in MATLAB environment and then control technique is applied on the SISO systems. For height control, multiple feedback loops are used; outer loop is chosen on the basis of primary control objective. Pitch is taken as inner loop and initially feedback gain is taken as 1 for simplicity, another feedback loop is taken from height and controller is placed in between the outer and inner loops. Controller gains can be calculated by Tyreus-Luyben Method in which the ultimate gain and the corresponding frequency are determined at the point where the system just starts oscillations [17]. Open loop root locus plot can be used to calculate these parameters. The ultimate Gain and Frequency are used to calculate controller gains.

5.2. Design of Sideslip and Speed Control Feedback Loops: Single feedback loop is used for sideslip control and similar for speed control. Controller gains are calculated for the system's appropriate performance. PI controllers are used for the control of all parameters of the non-holonomic time variant system. Lateral parameters can be controlled in similar 
manner. Control on both longitudinal and lateral parameters provide full control on the vehicle movements with six degrees of freedom.

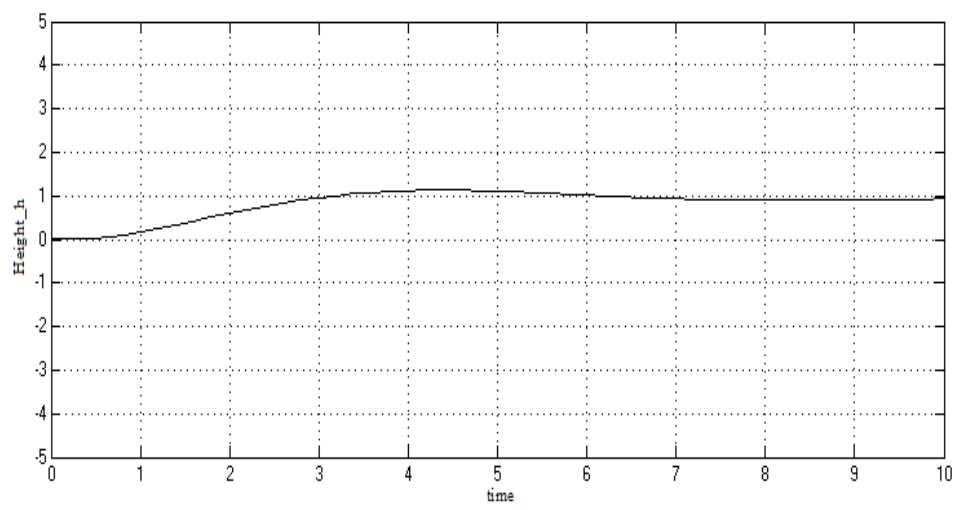

Figure.9 Closed Loop Sideslip Response of UAV

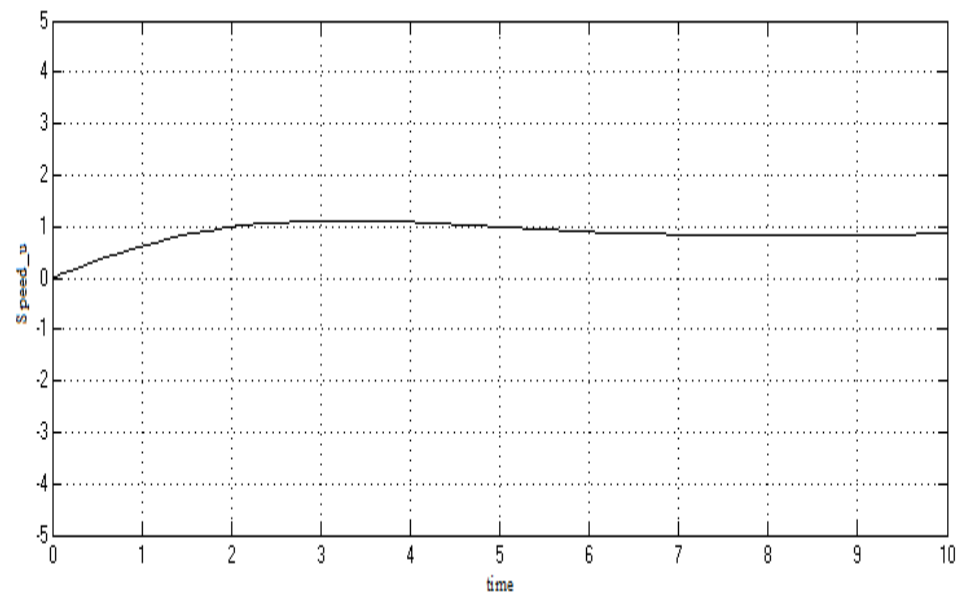

Figure 10. Closed Loop Height Response of UAV

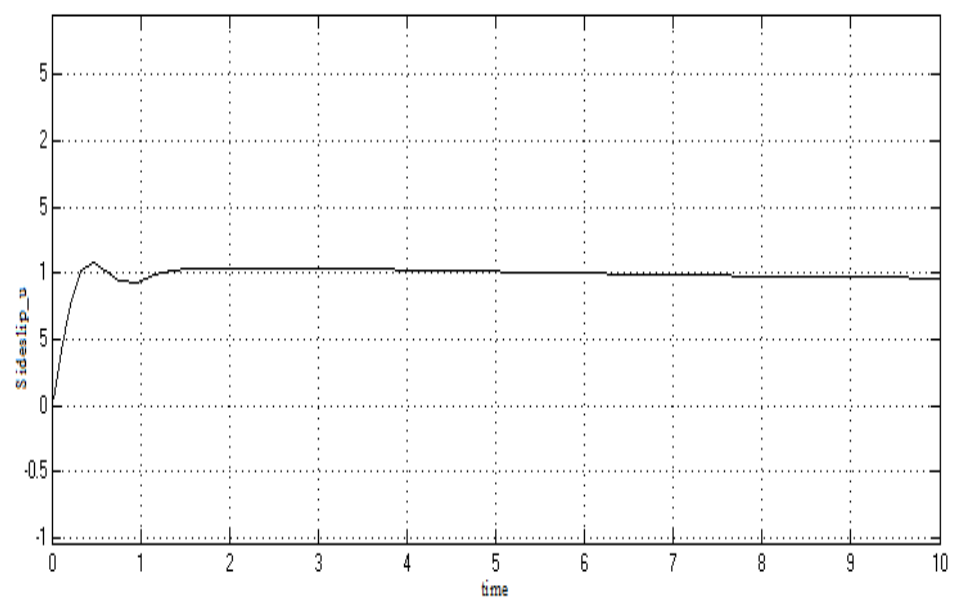

Figure.11 Closed Loop Speed Response of UAV 


\section{Design Results}

The PI controller gains for control of sideslip, height and speed of UAV are calculated using tyreus- Luyben tuning method and are tabulated in the Table 1.

Table 1. Controller Parameters

\begin{tabular}{|l|l|l|}
\hline Control Loop & Controller Type & Controller Gains \\
\hline Sideslip control loop & PI & $K_{p}=0.3579$ \\
& & $K_{\mathrm{i}}=4.0223$ \\
& & $K_{f}=0.9325$ \\
\hline Height control loop & PI & $K_{p}=0.0196$ \\
& & $K_{i}=0.0002$ \\
& & $K_{f}=0.9736$ \\
\hline Speed control loop & PI & $K_{p}=0.0048$ \\
& & $K_{i}=0.0031$ \\
\hline
\end{tabular}

Where:

$K_{p}$-Proportional Gain, $K_{i}$-Integral Gain, $K_{f}$-Feedback Gain

The closed loop responses of the UAV for sideslip, height and speed are shown in Figures 9, 10 and 1 respectively.

\section{Results and Conclusion}

In this paper, flight control design technique has been presented. Non-linear plant model has been simplified by linearization and then MIMO system has been converted into simple SISO systems. Keeping control objective in mind, multi-loop control technique and simple feedback control techniques have been applied. Simple PI controllers have been used to achieve control objectives. Longitudinal Autopilot is designed for a given flight conditions which provides outputs with negligible steady state errors and very small overshoots within few seconds of response time as shown in closed loop responses of UAV shown by Figures 9-11. Substantial improvement in system performance has been achieved and open loop unstable system has been efficiently controlled by closed loop control method.

\section{References}

[1] P. G. Fahlstrom and T. J. Gleason, "UAV System Introduction", $4^{\text {th }}$ edition, John Wiley and Sons aerospace series, (2012).

[2] J. M. Sullivan, "Evolution or Revolution? The rise of UAVs", IEEE Technology and Society Magazine, vol. 25, no. 3, (2006), pp. 43-49.

[3] D. McRuer, "A Flight Control Century: Triumphs of the Systems Approach," Journal of Guidance, Control and Dynamics, vol. 27, no. 2, (2003), pp. 161-173.

[4] Notes of US AF Test Pilot School Edwards AFB CA, "flying qualities phase, volume II", approved to public release, Distribution statement A, (1988) October. 
[5] L. Stevens and F. L. Lewis, "Aircraft Control and Simulation", 2nd ed., John Wiley \& Sons, (2003).

[6] M. V. Cook, "Flight Dynamics Principles", Elsevier Butterworth- Heinemann, (1997).

[7] H. Chao, Y. Cao and Y. Chen, "Autopilots for small fixed-wing unmanned air vehicle: A survey", IEEE International Conference on Mechatronics and Automation, (2007), pp. 3144-3149.

[8] I. H. Makhdoom and S.-Y. Qin, "Matlab-Based Flight Control Design Scheme for UAVs", Proceedings of the 8thWorld Congress on Intelligent Control and Automation, (2010) July 6-9, Jinan, China.

[9] M. Gopal, "Modern Control System Theory", 1st edition, New Age International, (1993).

[10] R. C. Nelson, "Flight stability and automatic control”, New York. McGraw-Hill, 2nd edition. (1998).

[11] MathWorks Inc., Matlab \& Simulink, (2011).

[12] C. Yun and X.-M. Li, "Aerodynamic Model Analysis and Flight Simulation Research of UAV Based on Simulink", Journal of Software Engineering and Applications, vol. 6, (2013), pp. 43-47.

[13] M. Chiaramonti and G. Mengali, "Control laws for a formation of autonomous flight vehicles", Aeronautical Journal, vol. 113, no. 1147, (2009), pp. 609-616.

[14] M.-L. Zhang, "Flight Control System”, Aviation Industry Press, Beijing, (1983).

[15] H. Mansur, M. Frye, B. Mettler and M. Montegut, "Rapid Prototyping and Evaluation of Control System Designs for Manned and Unmanned Applications", 56th Forum of the American Helicopter Society, Virginia Beach, VA, Mai, (2000).

[16] F. Liang, "Rapid development of UAV autopilot using Matlab/Simulink", Proceedings of the AIAA Modeling and Simulation Conference and Exhibit, Monterey, CA, (2002) August.

[17] D. Tyreus and W. L. Luyben, "Tuning PI controllers for integrator/dead time processes", Industrial and Engineering Chemistry Research, vol. 31, no. 11, (1992), pp. 2625-2628.

\section{Authors}

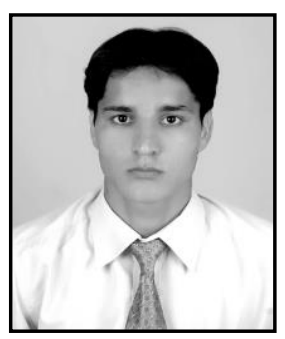

\section{Nilesh Kumar}

He did B.Tech in Electronics and Instrumentation from Kurukshetra, University India in 2008. Presently he is doing M. Tech in Instrumentation and Control from YMCA University of Science and Technology, Faridabad, India.

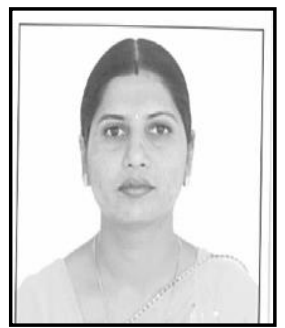

\section{Sheilza Jain}

She did B. Tech in Electronics and Communication Engineering from Kurukshetra University, India and $\mathrm{M}$. Tech with specialization in Control System from Maharishi Dayan and University, India. She did Ph.D in Control of Nano-positioning from Maharishi Dayan and University. Presently she is working as Assistant Professor in Electronics Engineering Department at YMCA UST Faridabad. Her interest area includes control system, analog circuits. 